\title{
Low level laser therapy as a modality to attenuate cytokine storm at multiple levels, enhance recovery, and reduce the use of ventilators in COVID-19
}

\author{
Soheila Mokmeli MD Anesthesiologist ${ }^{1}$, Mariana Vetrici MD, $\mathrm{PhD}^{2}$
}

\begin{abstract}
S Mokmeli MD, M Vetrici. Low level laser therapy as a modality to attenuate cytokine storm at multiple levels, enhance recovery, and reduce the use of ventilators in COVID-19. Can J Respir Ther 2020;56:25-31. doi: 10.29390/cjrt-2020-015.

The global pandemic COVID-19 is a contagious disease and its mortality rates ranging from $1 \%$ to $5 \%$ are likely due to acute respiratory distress syndrome (ARDS), and cytokine storm. A significant proportion of patients who require intubation succumb to the disease, despite the availability of ventilators and the best treatment practices. Researchers worldwide are in search of anti-inflammatory medicines in the hope of finding a cure for COVID-19. Low-level laser therapy (LLLT) has strong, anti-inflammatory effects confirmed by meta-analyses, and it may be therapeutic to ARDS. LLLT has been used for pain management, wound healing, and other health conditions by physicians, physiotherapists, and nurses worldwide for decades. In addition, it has been used in veterinary medicine for respiratory tract disease such as pneumonia. Laser light with low-power intensity is applied to the surface of the skin to produce local and systemic effects. Based on the clinical experience, peer-reviewed studies, and solid laboratory data in experimental animal models, LLLT attenuates cytokine storm at multiple levels and reduces the major inflammatory metabolites. LLLT is a safe, effective, low-cost modality without any side-effects that may be combined with conventional treatment of ARDS. We summarize the effects of LLLT on pulmonary inflammation and we provide a protocol for augmenting medical treatment in COVID-19 patients. LLLT combined with conventional medical therapy has the potential to prevent the progression of COVID-19, minimize the length of time needed on a ventilator, enhance the healing process, and shorten recovery time.
\end{abstract}

Key Words: COVID-19; ARDS; cytokine storm; low level laser therapy; anti-inflammatory; ventilator; photobiomodulation

\section{What is low level laser therapy?}

Low level laser therapy (LLLT) is also known as cold laser therapy or photobiomodulation therapy. LLLT utilizes visible light and infrared laser beams in the range of 450-1000 nm. Single wavelength or monochromatic light is emitted from a low-intensity laser diode $(<500 \mathrm{~mW})$. The light source is placed in contact with the skin, allowing the photon energy to penetrate tissue, where it interacts with various intracellular biomolecules to restore normal cell function and enhance the body's healing processes [1]. This contrasts with the thermal effects produced by the high-power lasers that are used in cosmetic and surgical procedures to destroy tissue [1], as mentioned in the PubMed Medical Subject Heading (MeSH) subheading for LLLT.

LLLT effects are not due to heat but rather to a photochemical reaction that occurs when a photoacceptor molecule within the cell absorbs a photon of light, becomes activated, and changes the cell's membrane permeability and metabolism. Presently, cytochrome c oxidase, opsins and their associated calcium channels, and water molecules have been identified as the main mediators of the photochemical mechanisms [2]. This leads to increased mRNA synthesis and cell proliferation. LLLT produces reactive oxygen species (ROS) in normal cells, but ROS levels are lowered when it is used in oxidatively stressed cells, like in animal models of disease. LLLT up-regulates antioxidant defenses and decreases oxidative stress [2].

Low-level lasers are a safe, noninvasive technology approved by both the US Food and Drug Administration and Health Canada for several chronic and degenerative conditions, temporary pain relief, cellulite treatment, body contouring, lymphedema reduction, hair growth, and chronic musculoskeletal injuries. LLLT increases microcirculation, lymphatic drainage, and cellular metabolism, thereby relieving many acute and chronic conditions.

The MeSH database in PubMed contains more than 7000 articles on LLLT. The effects of LLLT have been confirmed through several metaanalysis studies and include anti-inflammatory [3] and analgesic effects [4], tissue healing [5], treating tendinopathy [6], and improving lymphedema [7]. Recent lab and animal studies suggest LLLT is ready for clinical trials over myocardial infarction [5]. In 2010, a meta-analysis concluded that there was strong evidence of an anti-inflammatory effect of LLLT [3].

To date, published reports indicate that LLLT up-regulates antioxidant defenses and decreases ROS in oxidatively stressed cells and animal models of disease. The anti-inflammatory effect of LLLT directly addresses the main pathology of disorders such as musculoskeletal, lungs, wounds, brain, trauma, etc. LLLT reduces NF-kB, a protein complex that controls transcription of DNA, in pathological conditions. Reports have shown reductions in reactive nitrogen species and prostaglandins in various animal models [2].

LLLT has diverse effects [8]:

- reduces pain related to inflammation via dose-dependent reduction of prostaglandin E2, prostaglandin-endoperoxide synthase-2, IL-1, IL-6, TNFa, as well as the cellular influx of neutrophils, oxidative stress, edema, and bleeding;

${ }^{1}$ Canadian Optic and Laser Center (Training Institute), Victoria, BC, Canada

${ }^{2}$ Department of Biological Sciences, University of Lethbridge, Lethbridge, AB T1K 3M4, Canada

Correspondence: Soheila Mokmeli, Canadian Optic and Laser Center (Training Institute), 744A Lindsay Street, Victoria, BC V8Z 3E1, Canada. Tel.: +1 (250) 480-7868, E-mail: dr.mokmeli@yahoo.com Mariana A. Vetrici, Department of Biological Sciences, University of Lethbridge, 4401 University Drive, Lethbridge, AB T1K 3M4, Canada, Tel.: +1 (865) 888-3095, E-mail: marianavetrici@gmail.com

Published online at https://www.cjrt.ca on 23 July 2020 
- decreases edema and swelling by increasing lymphatic drainage;

- increases collagen and protein production, and cell proliferation;

- accelerates wound healing and scar formation;

- improves quality and tensile strength of tissue;

- stimulates nerve function and regeneration;

- accelerates bone regeneration and remineralization;

- reduces the pain threshold and enhances endorphins;

- washes inflammatory debris away from the injured site; and

- augments blood flow.

LLLT has been used in respiratory tract diseases since 1978. Empirical practice on over 1000 patients produced data pertaining to chronic pneumonia, acute pneumonia, asthma, and chronic bronchitis in children, adults, and elderly. Common findings include reduced chest pain and heaviness; normalization of respiratory function; improved blood, immunological, and radiological parameters; and shortened recovery times. In community-acquired pneumonia, intravenous LLLT of blood added to conventional treatment significantly promoted the bactericidal activity of neutrophils. In asthma, the addition of LLLT was more effective than medical treatment alone and it shortened the duration of treatment and recovered bronchial sensitivity to sympathomimetics [9-11]. In newborns with pneumonia, LLLT combined with conventional medical regimens optimized the treatment infectious and inflammatory diseases, reduced the incidence of complications, and shortened recovery periods [12].

LLLT is a well-known treatment modality in veterinary medicine. Upper and lower respiratory conditions in dogs and cats are common, and viral and bacterial infections are often highly contagious. Regardless of etiology, inflammation is the major pathology of these conditions. The addition of LLLT to conventional treatment alleviates symptoms and stimulates the healing process in tissues. General guidelines for the use of laser therapy in animals and protocols for specific conditions are published [13].

\section{The pathogenesis of COVID-19 in respiratory tract}

Coronaviruses are a large group of viruses that affect animals. In humans, they produce diseases such as the common cold, severe acute respiratory syndrome (SARS) and Middle East respiratory syndrome. The disease caused by the novel coronavirus, SARS-CoV-2, has been named COVID19 and the clinical manifestations range from asymptomatic to severe acute respiratory distress syndrome (ARDS) to death [14].

Respiratory viruses infect either the upper or lower airways. Typical upper-respiratory infections are milder, more contagious, and spread easily, whereas lower-respiratory infections spread much less frequently but are more severe and dangerous. SARS-CoV-2 appears to infect both upper and lower airways. It spreads while still limited to the upper airways, before traveling into the deeper respiratory tract and leading to severe symptoms [15].

SARS-CoV-2 attaches to a protein called angiotensin converting enzyme (ACE2), on the surface of cells in the respiratory tract. As SARSCoV-2 attacks the cells, dead cells flow down and block the airways with debris while the virus moves deeper into the lungs. Breathing becomes difficult because the lungs become clogged with dead cells and fluid. The immune system attacks the virus causing inflammation and fever. In severe cases, the immune system goes wild, causing more damage to the lungs than the actual virus. Blood vessels dilate to increase blood flow and become more permeable to maximize transport of chemical and cellular mediators the infection site. Inevitably, the lungs get filled with fluid. This exaggerated immune response is called cytokine storm and it leads to ARDS, fever, multiorgan failure, and death $[15,16]$.

During cytokine storm, the immune system attacks indiscriminately without clearing the specific targets. Cytokine storm also affects other organs, especially if people already have chronic diseases [15]. The severity of cytokine storm determines who is hospitalized and who will be treated in the intensive care unit (ICU). The classification of COVID-19 is summarized in Table 1 [17].

The morbidity and mortality of COVID-19 are due to excessive inflammatory cytokine production and immune hyperactivity. Alveolar macrophage activation and cytokine storm are the main pathogenesis of severe COVID-19. The pathological features include exudation and hemorrhage, epithelial injuries, infiltration of macrophages into the lungs, and fibrosis of lung tissue. The mucous plug with fibrinous exudate in the alveoli and the activation of alveolar macrophage are characteristic abnormalities [18, 19]. Chemical and genetic studies have shown that the pulmonary endothelium is a key component of the cytokine storm. Therefore, modulation of the involved cellular signaling pathways may have therapeutic effects [20, 21].

COVID-19 begins when SARS-CoV-2 uses ACE2 as the entry receptor for infection [22]. This induces ACE2 downregulation and shedding. Loss of ACE2 from the endothelium causes dysfunction of the renin-angiotensin system, and it enhances inflammation and vascular permeability. Shedding of ACE2 from the endothelium releases enzymatically active soluble ACE2 (sACE2), which is tightly linked to tumor necrosis factor alpha (TNF- $\alpha$ ) production in cell culture [23].

Multiple signaling pathways are activated during an immune response and cytokine storm. The $\mathrm{P} 2 \mathrm{X}$ purinoceptor 7 ( $\mathrm{P} 2 \mathrm{X} 7 \mathrm{r})$ is major

\section{TABLE 1}

The staging and classification of COVID-19 [17]

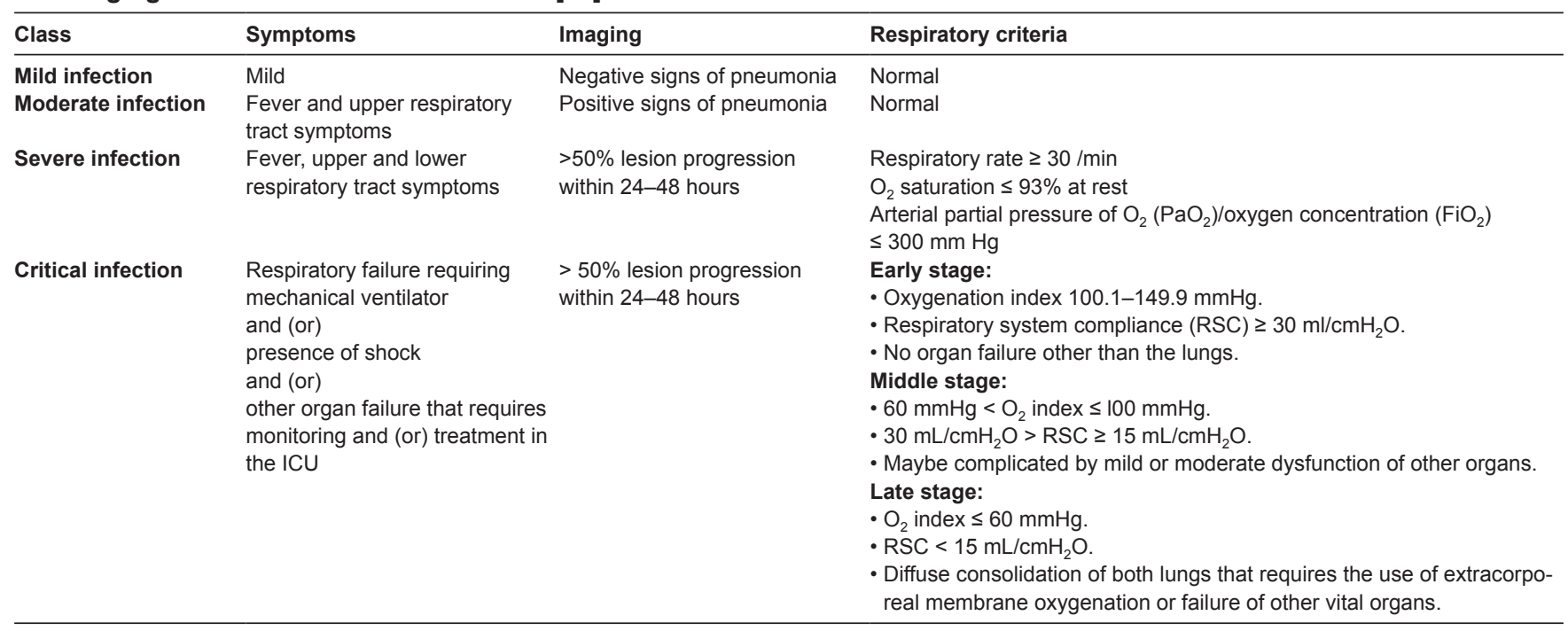

Note: A confirmed case is based on the epidemiological history (including cluster transmission), clinical symptoms (fever and respiratory symptoms), lung imaging, and results of SARS-CoV-2 nucleic acid detection and serum-specific antibodies [17]. 
factor involved in activation of the cytokine storm and lung pathology in response to viruses [24,25], infection, inflammation, hypoxia, or trauma [26]. P2X7r is an adenosine triphosphate (ATP) gated, nonselective cation channel, allowing $\mathrm{Ca}^{2+}$ and $\mathrm{Na}^{+}$influx and $\mathrm{K}^{+}$efflux. Extracellular ATP plays a central role in apoptotic cell death [27], the induction of inflammation [28], and mitochondrial failure in monocytes [29]. P2X7r mediates ATP-induced cell death in different cells and it promotes assembly and release of proinflammatory interleukins (IL-1 $\beta$ and IL-18) from immune cells after exposure to lipopolysaccharide and ATP [27]. $\mathrm{P} 2 \mathrm{X} 7 \mathrm{r}$ is constitutively expressed in many cells, including respiratory epithelial cells and most immune cells like neutrophils, monocytes, macrophages, dendritic, natural killer, B and T lymphocytes [27].

Studies stratified COVID-19 patients as: (i) severe symptoms and ICU admission and (ii) mild and moderate symptoms requiring hospitalization but not ICU $[17,19]$. The severe patients have significantly higher levels of plasma pro-inflammatory factors (IL-2, IL-7, IL-10, GSCF, IP-10, MCP-1, MIP1A, TNF- $\alpha$ ) [19] and (IL-2, IL-6, IL-10, TNF- $\alpha$ ) [18] than non-ICU patients, and they were likely in cytokine storm [17, 19]. These findings justify the use of IL-6 receptor antagonists [18, 19]; however, a therapy to reduce inflammation at multiple levels, such as LLLT, could be more successful in controlling the unbalanced immune response (Figure 1).

\section{The effects of LLLT on pulmonary inflammation}

LLLT is effective against cytokine storm and ARDS while promoting healing and tissue regeneration. Experimental and animal models of pulmonary disease and infection have revealed multiple cellular and molecular effects, which are both local and systemic. LLLT reduces inflammation without impairing lung function in acute lung injuries and is a promising therapeutic approach for lung inflammatory diseases such as Chronic obstructive pulmonary disease [26].

In murine models of acute inflammation of the airways and lungs, transcutaneous LLLT delivered over the trachea decreases pulmonary microvascular leakage [30, 31], IL-1b levels [26, 30], IL-6 [26, 32], MIP-2 mRNA expression [30], and intracellular ROS production [24]. LLLT produces anti-inflammatory effects on tracheal hyperactivity, and reduces neutrophil influx [26, 30, 32-34] by inhibiting COX-2-derived metabolites [33]. In ARDS, LLLT elevates cyclic adenosine monophosphate [32, 34], a signaling molecule that stimulates IL-10 and G-CSF expression and blocks TNF-a and MIP-1. LLLT also reduces TNF-a levels in bronchoalveolar lavage fluid and alveolar macrophages [26, 31-34]. In hemorrhagic lesions of the lungs, LLLT significantly reduces the hemorrhagic index and myeloperoxidase activity, to levels comparable to Celecoxib [35].

LLLT contributes to the resolution of inflammation by upregulating IL-10 and downregulating P2X7r. LLLT changes the profile of inflammatory cytokines and elevates IL-10 [26, 31, 36], known as human cytokine synthesis inhibitory factor, in the lung and abolishes lung inflammation via a reduction of inflammatory cytokines and mast cell degranulation [31]. LLLT decreases collagen deposition as well as the expression of the P2X7r [26].

LLLT contributes to healing by promoting apoptosis of inflammatory cells while suppressing apoptotic pathways in lung tissue. In a model of acute lung injury, LLLT reduced DNA fragmentation and apoptotic pathways via increased B-cell-lymphoma-2 (Bcl-2), the key regulator of the intrinsic or mitochondrial pathway for apoptosis, in alveolar epithelial cells while promoting DNA fragmentation in inflammatory cells [37]. In pulmonary idiopathic fibrosis, LLLT inhibits pro-inflammatory cytokines and increases expression of proliferating cell nuclear antigen [38], attenuates airway remodeling by balancing pro- and anti-inflammatory cytokines in lung tissue, and inhibiting fibroblast secretion of the pro-fibrotic cytokines [36].

LLLT provides synergy in combination with medical treatment. It has a synergic anti-inflammatory action over alveolar macrophages pretreated with $\mathrm{N}$-acetyl cysteine, an effective oral medicine for coughs and some lung conditions [39]. The synergic effects of LLLT combined with conventional treatments were reported on over 1000 patients in Russian studies [9-11].

\section{FIGURE 1}

The effects of SARS-CoV-2 on alveolar cell and cytokine storm.

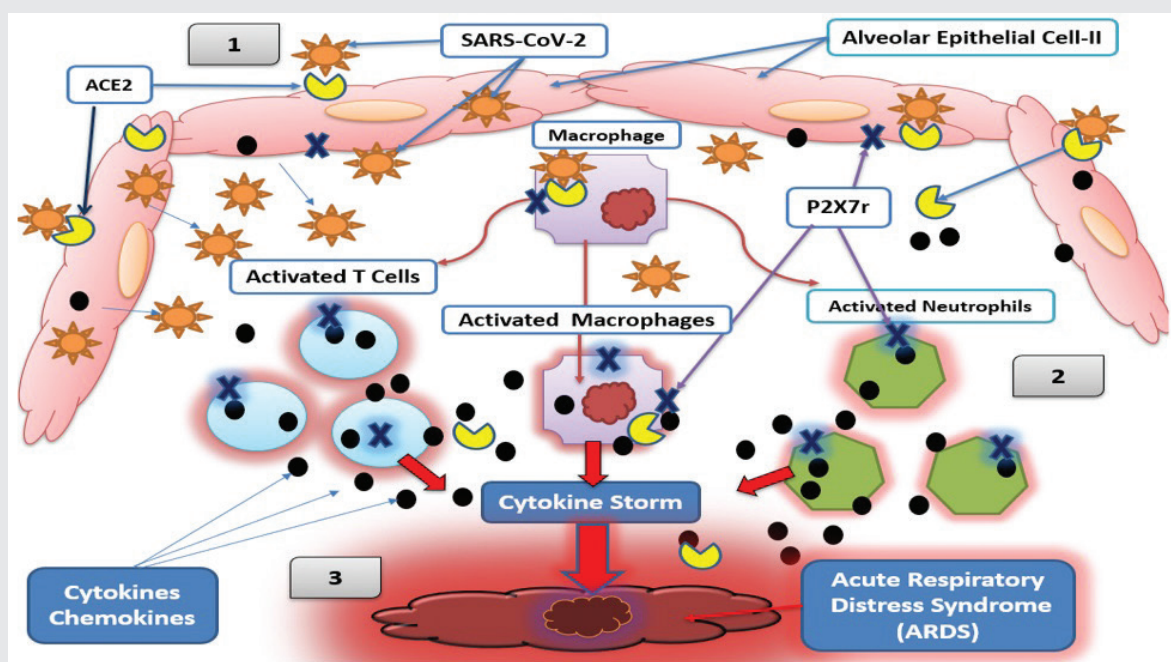

1 - SARS-CoV-2 particles attack lung epithelial cells, immune cells and alveolar macrophage by attaching to ACE-2 to generate more viruses and release cytokines/chemokines (Inflammatory Elements). SARS-CoV-2 Induces ACE2 downregulation and suspending the ACE2. Activation of P2X7 receptors by extracellular ATP plays a key role in the induction of inflammation.

2 - Cytokine/chemokine-activated macrophages and virally infected T cells and other neutrophils lead to an extensive immune response and the initiation of cytokine storm.

3 - Released chemokines load more inflammatory cells from blood vessels into the site of inflammation, and this amplifies cytokine storm and leads to Acute Respiratory Distress Syndrome (ARDS). 
Extended time on ventilators causes lung injury but LLLT minimizes this side effect. In experimental models of ventilator-induced lung injury (VILI), LLLT following VILI resulted in lower injury scores, decreased total cell count and neutrophil count in bronchoalveolar lavage, and reduced alveolar neutrophil infiltration. LLLT in an experimental model of VILI in rats demonstrated the anti-inflammatory effect via decreased lung injury scores and lower counts of neutrophils in alveolar, interstitial, and bronchial lavage [39] (Figure 2).

Evidence from the literature supports the use of LLLT for the treatment of COVID-19.

- It has significant anti-inflammatory effects confirmed by metaanalyses. Eleven cell studies, 27 animal studies, and another six animal studies for drug comparisons and LLLT interactions verified that there is strong evidence of an anti-inflammatory effect of LLLT. The scale of the anti-inflammatory effect is not significantly different than non-steroidal anti-inflammatory drugs, but it is slightly less than glucocorticoid steroids [3].

- It has diverse applications and effects confirmed through several meta-analysis studies include analgesia [4], tissue healing [5], treating tendinopathy [6], and improved lymphedema [7].

- LLLT is approved by the US FDA and Health Canada for several chronic and degenerative conditions, temporary pain relief, cellulite treatment, body contouring, lymphedema reduction, and hair growth. It has been used in veterinary medicine for upper and lower respiratory conditions in dogs and cats [13].

- It has been used for human respiratory tract disease. Empirical use on over 1000 patients produced data pertaining to chronic pneumonia, acute pneumonia, asthma, and chronic bronchitis in children, adults, and the elderly [9-12]. Light therapy and LLLT has been mentioned as a potential treatment for pandemic coronavirus infections [40].

- The anti-inflammatory effect of LLLT in lung inflammation is confirmed in at least 14 experimental animal studies. LLLT attenuates cytokine storm at multiple levels and reduces the major inflammatory metabolites such as IL- 6 and TNF- $\alpha$. IL- 6 antagonists are being investigated for treating COVID-19 but LLLT reduces the production of IL-6, as well as other chemokines and metabolites [26-39, 41].

- There are US FDA and Health Canada approved laser machines for pain management, lymphedema after breast cancer surgery, and cellulite treatments that can be used and set to treat lung inflammation.

- LLLT is an affordable modality compared with other treatments and medicines like IL-6 antagonists. LLLT is a safe, effective, lowcost modality without any reported side-effects compared with other approaches. A laser machine costs Can $\$ 35,000.00$ $200,000.00$, and each machine can fully treat 20,000 patients for COVID-19. In comparison, an IL-6 antagonist costs US $\$ 1000.00$ per injection, and each patient would need 3-6 injections for complete COVID-19 treatment. Treating 20,000 patients would cost US\$ 60,000,000.00-US\$ 120,000,000.00.

Based on this information, LLLT will accelerate recovery from COVID19 and will get patients off ventilator support and out of the ICU more rapidly. This could significantly decompress our severely overburdened health care systems.

\section{Therapeutic technique and dosage of LLLT}

Laser dose is the amount of energy delivered per second per $\mathrm{cm}^{2}$. The effect of laser therapy is related to the amount of laser energy per $\mathrm{cm}^{2}$. The ArndtSchultz Law is considered the standard to describe the dose dependent effects of LLLT [42]. The minimum therapeutic dose for a bio-stimulatory effect for red and infrared laser is $0.01 \mathrm{~J} / \mathrm{cm}^{2}$ while for ultraviolet, blue, green laser it is $0.001 \mathrm{~J} / \mathrm{cm}^{2}$. LLLT has a noticeable biphasic dose response. The effective stimulation dose is $1 \mathrm{~J} / \mathrm{cm}^{2}$ on the target tissue. Doses greater than $10 \mathrm{~J} / \mathrm{cm}^{2}$ produces inhibitory effects. The inhibitory effects are used in conditions requiring inhibition and suppression [2].

\section{FIGURE 2}

The effects of SARS-CoV-2 versus LLLT on cytokine storm and lung tissue.

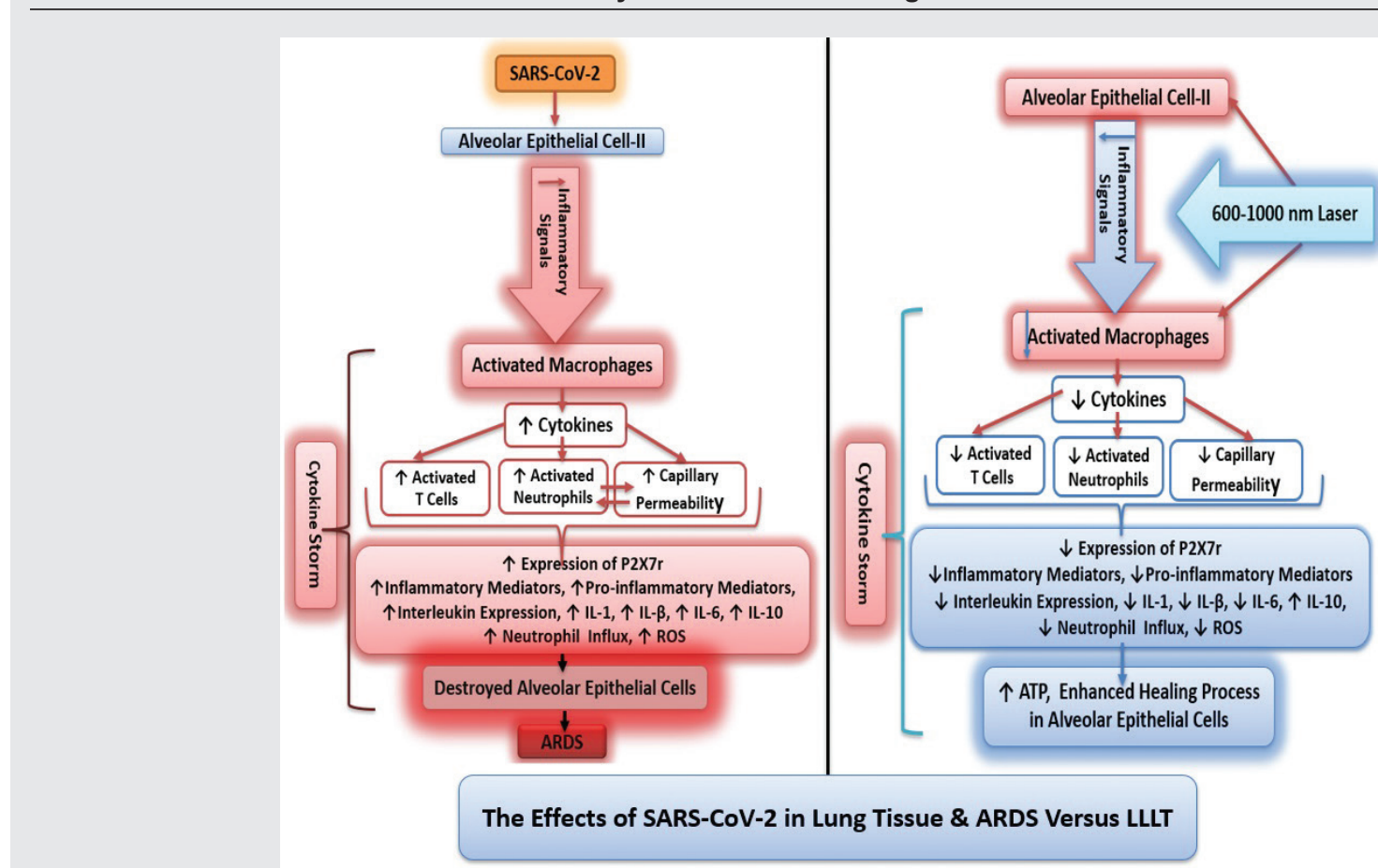


Therapeutic protocol: early phase of COVID-19: (Figure 3, Table 2) Laser parameters:

- Laser type: infrared laser (780-900 nm), or red laser (630-660 nm)

- Average power: $50-100 \mathrm{~mW}$

- Dose: $4-6 \mathrm{~J} / \mathrm{cm}^{2}$

- Area: $10 \mathrm{~cm}^{2}$

- Time: 1-2 minutes/ $\mathrm{cm}^{2}$

- Sessions: 3-8 once-daily sessions

Laser probe positions:

- Intranasal: 2 minutes, noncontact technique

- Over right and left tonsils: transcutaneous (place laser over the skin)

- Over the trachea: transcutaneous

- Over the veins in the cubital areas: transcutaneous blood laser therapy, $10-15$ minutes

Therapeutic protocol: medium-severe phase of COVID-19: (Figure 3, Table 3)

Laser parameters:

- Laser type: infrared laser (780-900 nm) or red laser (630-660 nm)

- Average power: $50-100 \mathrm{~mW}$

- Dose: $6-10 \mathrm{~J} / \mathrm{cm}^{2}$

- Area: $10 \mathrm{~cm}^{2}$

- Time: 2-3 minutes/ $\mathrm{cm}^{2}$

- Sessions: 3-10 once-daily sessions

Laser Probe Positions:

- Over the lungs: bilaterally over apical, middle, and lower lobes and front and back of thorax, transcutaneous over the intercostal spaces

- Over the trachea: transcutaneous

- Over the bronchus: upper mediastinal area, transcutaneous
- Over right and left tonsils: transcutaneous

- Over the veins in the cubital areas: transcutaneous blood laser therapy; $10-15$ minutes

Contraindications and side effects of LLLT [42]

Although LLLT is safe and noninvasive and there are no reports of mutagenicity, genotoxicity, or carcinogenicity of LLLT after 60 years of its use. However, there are some contraindications:

- work over the site of tumors and cancer;

- benign tumors with possibility of converting to malignant tumors;

\section{TABLE 2}

Therapeutic protocol: Early phase of COVID-19

\begin{tabular}{ll}
\hline Laser system parameters & \\
\hline Wavelengths & Infrared laser $(780-900 \mathrm{~nm})$, or red \\
& laser $(630-660 \mathrm{~nm})$ \\
Average power & $50-100 \mathrm{~mW}$ \\
Dose & $4-6 \mathrm{~J} / \mathrm{cm}^{2}$ \\
Area & $10 \mathrm{~cm}^{2}$ \\
Sessions & $3-8$ once-daily sessions
\end{tabular}

\section{Laser probe positions}

Intranasal: Noncontact technique

1 minute $/ \mathrm{cm}^{2}(100 \mathrm{~mW})$

2 minutes $/ \mathrm{cm}^{2}(50 \mathrm{~mW})$

Over right and left tonsils Transcutaneous

1 minute $/ \mathrm{cm}^{2}(100 \mathrm{~mW}) \quad$ (place laser over the skin)

2 minutes $/ \mathrm{cm}^{2}(50 \mathrm{~mW})$

Over the trachea

1 minute $/ \mathrm{cm}^{2}(100 \mathrm{~mW})$

2 minutes $/ \mathrm{cm}^{2}$ (50 mW)

Over the veins in the cubital areas Transcutaneous blood laser therapy

8 minute $/ \mathrm{cm}^{2}(100 \mathrm{~mW})$

15 minutes $/ \mathrm{cm}^{2}(50 \mathrm{~mW})$

\section{FIGURE 3}

\section{LLLT for COVID-19.}

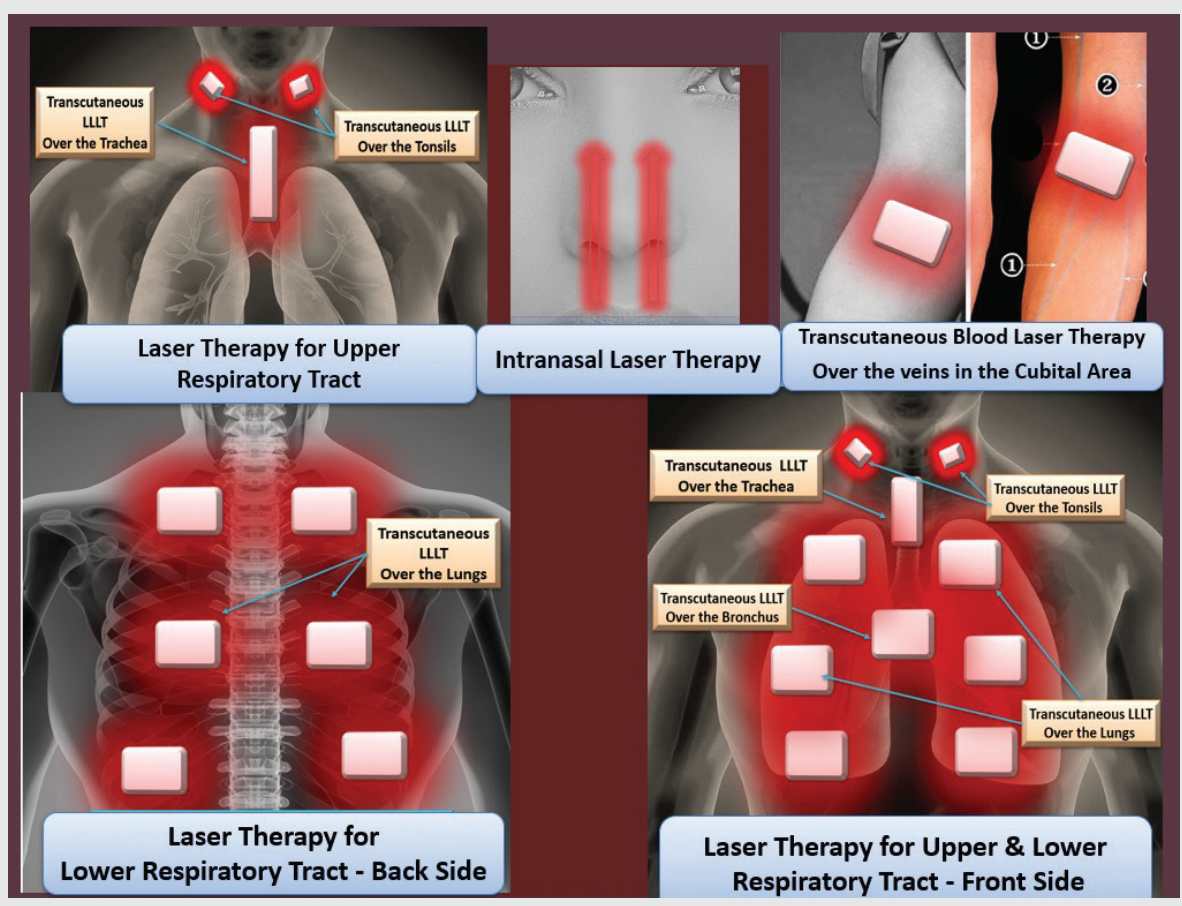




\section{TABLE 3}

Therapeutic protocol: medium-severe phase of COVID-19

\begin{tabular}{ll}
\hline Laser system parameters & \\
\hline Wavelengths & Infrared laser $(780-900 \mathrm{~nm})$, or red laser \\
& $(630-660 \mathrm{~nm})$ \\
Average power & $50-100 \mathrm{~mW}$ \\
Dose & $6-10 \mathrm{~J} / \mathrm{cm}^{2}$ \\
Area & $10 \mathrm{~cm}^{2}$ \\
Sessions & $3-10$ once-daily sessions \\
\hline
\end{tabular}

Laser probes positions

Intranasal: Noncontact technique

1 minute $/ \mathrm{cm}^{2}(100 \mathrm{~mW})$

2 minutes $/ \mathrm{cm}^{2}(50 \mathrm{~mW})$

Over right and left tonsils

1 minute $/ \mathrm{cm}^{2}(100 \mathrm{~mW})$

2 minutes $/ \mathrm{cm}^{2}(50 \mathrm{~mW})$

Over the trachea

1 minute $/ \mathrm{cm}^{2}(100 \mathrm{~mW})$

2 minutes $/ \mathrm{cm}^{2}(50 \mathrm{~mW})$

Over the veins in the cubital

areas

8 minute $/ \mathrm{cm}^{2}(100 \mathrm{~mW})$

15 minutes $/ \mathrm{cm}^{2}(50 \mathrm{~mW})$

Over the lungs

$1: 30-2$ minute $/ \mathrm{cm}^{2}(100 \mathrm{~mW})$

2-3 minutes $/ \mathrm{cm}^{2}(50 \mathrm{~mW})$

Over the bronchus

$1: 30-2$ minute $/ \mathrm{cm}^{2}(100 \mathrm{~mW})$

$2-3$ minutes $/ \mathrm{cm}^{2}(50 \mathrm{~mW})$

- the first 3 months of pregnancy (in the second and third trimesters, avoid work on abdominal and spine area); and

- light sensitivity conditions.

Precautions [42]

- epiphyseal line in children;

- glands: avoid ovaries, testes;

- in patients with severe end organ damage: heart, kidney, liver, and lung;

- epilepsy: the possibility of nerve discharge is increased in LLLT, especially with low-frequency protocols, 5-10 HZ.

\section{Side effects of LLLT}

Optical side effects

Because of the high intensity of lasers and the absorption of its wavelengths by different parts of ocular system, there is a possibility of damage to the eyes. It is important to use protective glasses that can absorb the specific wavelength. Protective glasses for each wavelength are different; therefore, choose the protective goggles specified for each wavelength. Both therapists and clients should wear protective goggles [42].

Early sense of healing

The analgesic effect of laser manifests earlier than its healing effect, and the patients feel better because of this, but the actual tissue damage has not yet healed. Patients feel relaxed and more energetic because the pain is gone. However, they must allow enough time for recovery [42].

\section{Fatigue and tiredness}

Fatigue is the most common symptom following LLLT. This is due to hormonal and metabolite changes after laser therapy that increase expression natural pain killers like endorphins and enkephalins. These metabolites induce relaxation and sleepiness [42].

\section{Low blood pressure and dizziness}

Very rarely, when the treated area is close to large blood vessels, a patient may experience a temporary drop in the blood pressure and orthostasis. This is due to vasodilatation and increased circulation to the limbs. To avoid dizziness, it is recommended that patients drink fluids before LLLT, and then wait for a few minutes before getting up from the supine position [42].

\section{CONCLUSION}

COVID-19 is potentially lethal because of cytokine storm and ARDS. Although most patients who contract COVID-19 may recover at home, a significant proportion require hospitalization and (or) ICU treatment. Many of the patients that are placed on ventilators succumb to the disease despite the best treatment practices. Often, patients are maintained on ventilators for longer than expected, and this may contribute to ventilator induced lung injury while depleting the patient's convalescent resources. Modulation of inflammatory factors and a boost to healing are necessary to help patients come off the ventilators. LLLT is a safe and noninvasive modality that has been used for decades in pain management, wound healing, and health conditions including diseases of the respiratory tract. LLLT was combined successfully with standard medical care to optimize response to treatments, reduce inflammation, promote healing, and accelerate recovery times. Scientific evidence shows that LLLT attenuates the inflammatory cytokines and chemokines in cytokine storm at multiple levels. In addition, LLLT promotes apoptosis of inflammatory cells and protects alveolar cells from damage. These findings suggest that LLLT is a feasible modality in the treatment of ARDS. LLLT can be added to the conventional treatment in COVID-19 at different stages of the disease. Because of its anti-inflammatory effect, and ability to shorten recovery times, LLLT can reduce the need of ventilators in the healing process. Clinical trials are necessary to objectively evaluate the effect of LLLT on COVID-19 treatment and recovery.

\section{Contributors}

Soheila Mokmeli and Mariana Vetrici contributed to the conception and design of the work.

\section{Competing interests}

All authors have completed the ICMJE uniform disclosure form at www. icmje.org/coi_disclosure.pdf and declare: no financial relationships with any organizations that might have an interest in the submitted work in the previous 3 years; no other relationships or activities that could appear to have influenced the submitted work.

\section{Ethical approval}

Informed consent was obtained from all participants.

\section{REFERENCES}

1. Cotler HB, Chow RT, Hamblin MR, Carroll J. The use of Low Level Laser Therapy (LLLT) for musculoskeletal pain. MOJ Orthop Rheumatol 2015;2(5):00068. doi: 10.15406/mojor.2015.02.00068.

2. Hamblin MR. Mechanisms and applications of the anti-inflammatory effects of photobiomodulation. AIMS Biophys 2017;4(3):337-61. doi: 10.3934/biophy.2017.3.337.

3. Bjordal JM, Lopes-Martins RAB, Iversen VV. The anti-inflammatory mechanism of low level laser therapy and its relevance for clinical use in physiotherapy. Medicine 2010. 15: 286-293 Corpus ID: 27687471. doi: 10.1179/1743288X10Y.0000000001.

4. Jang $\mathrm{H}$, Lee $\mathrm{H}$. Meta-analysis of pain relief effects by laser irradiation on joint areas. Photomed Laser Surg 2012 Aug;30(8):405-17. doi: 10.1089/ pho.2012.3240.

5. Woodruff LD, Bounkeo JM, Brannon WM, et al. The efficacy of laser therapy in wound repair: a meta-analysis of the literature. Photomed Laser Surg 2004;22(3):241. Available at: https://www.ncbi.nlm.nih.gov/ pubmed/15315732 [Accessed 1 April 2020.]

6. Tumilty S, Munn J, McDonough S, Hurley DA, Basford JR, Baxter GD. Low level laser treatment of tendinopathy: a systematic review with meta-analysis. Photomed Laser Surg 2010;28(1):3-16. Available at: https://search.pedro.org.au/search-results/record-detail/25413 [Accessed 1 April 2020.]

7. Smoot B, Chiavola-Larson L, Lee J, Manibusan H, Allen DD. Effect of low-level laser therapy on pain and swelling in women with breast cancer-related lymphedema: a systematic review and meta-analysis. J Cancer Survivorship 2014;9(2). doi: 10.1007/s11764-014-0411-1. 
8. Simunovic Z. (chapter XIV); Gerard Danhof (chapter V); Laser in medicine and dentistry. European Medical Laser Association, CIP, University Library of Rijekam Croatia. ISBN: 953-6059-30-4; 2000

9. Amirov NB. Parameters of membrane permeability, microcirculation, external respiration, and trace element levels in the drug-laser treatment of pneumonia [Article in Russian]. Ter Arkh 2002;74(3):40-3.

10. Derbenev VA, Mikhailov VA, Denisov IN. Use of low-level laser therapy (LLLT) in the treatment of some pulmonary diseases: ten-year experience. Proc SPIE 2000;4166:323-5. doi: 10.1117/12.389506.

11. Ostronosova NS. Outpatient use of laser therapy in bronchial asthma. TerArkh 2006;78(3):414.

12. Erkinovna TB, Tulkunovna MH. Efficacy of laser therapy in infants with infectious-inflammatory respiratory diseases. АКРОНИМ: European Science Review; 2006. ISSN: Печатный: 2310-5577. Available at: https:// cyberleninka.ru/article/n/efficacy-of-laser-therapy-in-infants-with-infectious-inflammatory-respiratory-diseases [Accessed 1 April 2020.]

13. Arza RA. The chapter of upper and lower respiratory conditions: photobiomodulation. Laser therapy in veterinary medicine. 2017. doi: 10.1002/9781119220190.ch14.

14. Wu Z, McGoogan JM. Characteristics of and important lessons from the coronavirus disease 2019 (COVID-19) outbreak in China: summary of a report of 72314 cases from the Chinese Center for Disease Control and Prevention. JAMA 2020. doi: 10.1001/jama.2020.2648.

15. Yong E. Why the coronavirus has been so successful? Science 2020. Available at: https://www.theatlantic.com/science/archive/2020/03/ biography-new-coronavirus/608338/ [Accessed 1 April 2020.]

16. Mehta P, McAuley DF, Brown M, et al. COVID-19: consider cytokine storm syndromes and immunosuppression. Lancet 2020;395(10229): P1033-4. doi: 10.1016/S0140-6736(20)30628-0.

17. Liang T. According to clinical experience; Handbook of COVID-19 prevention and treatment. Zhejiang University School of Medicine, Hangzhou; 2020.

18. Wang C, Xie J, Zhao L, et al. Alveolar macrophage activation and cytokine storm in the pathogenesis of severe COVID-19. Preprint from Research Square; 2020. doi: 10.21203/rs.3.rs-19346/v1.

19. Huang C, Wang Y, Li X, et al. Clinical features of patients infected with 2019 novel coronavirus in Wuhan. Lancet China 2020. doi: 10.1016/ S0140-6736(20)30183-5.

20. Damjanovic D, Small CL, Jeyanathan M, et al. Immunopathology in influenza virus infection: uncoupling the friend from foe. 2012 Clin Immunol 144:5769. doi: 10.1016/j.clim.2012.05.005.

21. Teijaro JR, Walsh KB, Cahalan S, et al. Endothelial cells as regulators of cytokine storms during influenza infection. Cell 2011;146:980e91. Available at: https://thorax.bmj.com/content/thoraxjnl/early/2011/ 12/14/thoraxjnl-2011-201437.full.pdf [Accessed 29 March 2020.]

22. Zhou P, Yang XL, Wang XG, et al. A pneumonia outbreak associated with a new coronavirus of probable bat origin. Nature 2020. doi: 10.1038/s41586-020-2012-7.

23. Fu Y, Cheng Y, Wu Y. Understanding SARS-CoV-2-mediated inflammatory responses: from mechanisms to potential therapeutic tools. Virol Sin 2020. doi: 10.1007/s12250-020-00207-4.

24. Leyva-Grado VH, Ermler ME, Schotsaert M, et al. Contribution of the purinergic receptor P2X7 to development of lung immunopathology during influenza virus infection. mBio 2017. doi: 10.1128/mBio.00229-17.

25. Rosli S, Kirby FJ, Lawlor KE, et al. Repurposing drugs targeting the P2X7 receptor to limit hyperinflammation and disease during influenza virus infection. Br J Pharmacol 2019. doi: 10.1111/bph.14787.

26. da Cunha Moraes G, Vitoretti LB, de Brito AA, et al. Low-level laser therapy reduces lung inflammation in an experimental model of chronic obstructive pulmonary disease involving P2X7 receptor. Oxid Med Cell Longev 2018 Mar 4;2018:6798238. doi: 10.1155/2018/6798238.

27. Kawano A, Tsukimoto M, Noguchi T, et al. Involvement of P2X4 receptor in P2X7 receptor-dependent cell death of mouse macrophages. Biochem Biophys Res Commun 2012;419(2):374-80. doi: 10.1016/j. bbrc.2012.01.156.
28. He Y, Taylor N, Fourgeaud L, Bhattacharya A. The role of microglial P2X7: modulation of cell death and cytokine release. J Neuroinflammation 2017. doi: 10.1186/s12974-017-0904-8.

29. Martínez-García JJ, Martínez-Banaclocha H, Angosto-Bazarra D, et al. $\mathrm{P} 2 \mathrm{X} 7$ receptor induces mitochondrial failure in monocytes and compromises NLRP3 inflammasome activation during sepsis. Nat Commun 2019;10:2711. doi: 10.1038/s41467-019-10626-x.

30. Aimbire F, Ligeiro de Oliveira AP, Albertini R et al. Low level laser therapy (LLLT) decreases pulmonary microvascular leakage, neutrophil influx and IL-1beta levels in airway and lung from rat subjected to LPS. induced inflammation. Inflammation 2008;31(3):189-97. doi: 10.1007/ s10753-008-9064-4.

31. Miranda da Silva C, Peres Leal M, Brochetti RA, et al. Low level laser therapy reduces the development of lung inflammation induced by formaldehyde exposure. PLoS One 2015 Nov 16;10(11):e0142816. doi: 10.1371/journal.pone.0142816.

32. Oliveira MC Jr, Greiffo FR, Rigonato-Oliveira NC, et al. Low level laser therapy reduces acute lung inflammation in a model of pulmonary and extrapulmonary LPS-induced ARDS. J Photochem Photobiol B 2014:134:57-63. doi: 10.1016/j.jphotobiol.2014.03.021.

33. Aimbire F, Albertine R, de Magalhães RG, et al. Effect of LLLT Ga-Al-As $(685 \mathrm{~nm})$ on LPS-induced inflammation of the airway and lung in the rat. Lasers Med Sci 2005;20(1):11-20. doi: 10.1007/ s10103-005-0339-9.

34. de Lima FM, Moreira LM, Villaverde AB, Castro-Faria-Neto HC, Aimbire F. Low-level laser therapy (LLLT) acts as cAMP-elevating agent in acute respiratory distress syndrome. Lasers Med Sci 2011 May 24;26(3):389-400. doi: 10.1007/s10103-010-0874-x.

35. Aimbire F, Lopes-Martins RA, Albertini R, et al. Effect of low-level laser therapy on hemorrhagic lesions induced by immune complex in rat lungs. Photomed Laser Surg 2007 Apr;25(2):112-7. doi: 10.1089/ pho.2006.1041.

36. de Brito AA, da Silveira EC, Rigonato-Oliveira NC, et al. Low-level laser therapy attenuates lung inflammation and airway remodeling in a murine model of idiopathic pulmonary fibrosis: relevance to cytokines secretion from lung structural cells. J Photochem Photobiol B 2020 Jan;203:111731. doi: 10.1016/j.jphotobiol.2019.111731.

37. Sergio LPDS, Thomé AMC, Trajano LADSN, et al. Photobiomodulation prevents DNA fragmentation of alveolar epithelial cells and alters the mRNA levels of caspase 3 and Bcl-2 genes in acute lung injury. Photochem Photobiol Sci 2018 Jul 11;17(7):975-83. doi: 10.1039/ c8pp00109j.

38. Rigonato-Oliveira NC, Brito AA, Moraes G, et al. Effect of low-level laser therapy in a model of pulmonary idiopathic fibrosis. Eur Respir J 2015;46:PA945. doi: 10.1183/13993003.congress-2015.PA945; Available at: https://erj.ersjournals.com/content/46/suppl_59/PA945 [Accessed 21 March 2020.]

39. Mafra de Lima F, Balbin Villaverde A, Albertini R, Ligeiro de Oliveira AP, Castro Faria Neto HC, Aimbire F. Low-level laser therapy associated to $\mathrm{N}$-acetylcysteine lowers macrophage inflammatory protein-2 (MIP-2) mRNA expression and generation of intracellular reactive oxygen species in alveolar macrophages. Photomed Laser Surg 2010;28(6):763-71. doi: 10.1089/pho.2009.2638.

40. Enwemekaa CS, Bumahab VV, Masson-Meyersc DS. Light as a potential treatment for pandemic coronavirus infections: a perspective. J Photochem Photobiol B 2020;111891. doi: 10.1164/ajrccm-conference.2019.199.1_MeetingAbstracts.A6152.

41. Lucinda LMF, Fazza TF, Fonseca LMC, et al. Effect of low-level laser therapy on experimental model of ventilator-induced lung injury. American Thoracic Society; International Conference Abstracts; B31. Acute lung injury \& ARDS: transitional and mechanistic studies; 2019 May 22.

42. Tuner J, Hode L. Laser therapy. Clinical practice and scientific background. BookGra" ngesberg, Sweden: Prima Books AB; 2002. ISBN: 91-631-1344-9 